\title{
'Residual diversity estimates' do not correct for sampling bias in palaeodiversity data
}

Article

Accepted Version

Sakamoto, M., Venditti, C. and Benton, M. J. (2017) 'Residual diversity estimates' do not correct for sampling bias in palaeodiversity data. Methods in Ecology and Evolution, 8 (4). pp. 453-459. ISSN 2041-210X doi:

https://doi.org/10.1111/2041-210X.12666 Available at https://centaur.reading.ac.uk/67779/

It is advisable to refer to the publisher's version if you intend to cite from the work. See Guidance on citing.

Published version at: http://dx.doi.org/10.1111/2041-210X.12666

To link to this article DOI: http://dx.doi.org/10.1111/2041-210X.12666

Publisher: Wiley-Blackwell

All outputs in CentAUR are protected by Intellectual Property Rights law, including copyright law. Copyright and IPR is retained by the creators or other copyright holders. Terms and conditions for use of this material are defined in the End User Agreement.

www.reading.ac.uk/centaur 
Central Archive at the University of Reading

Reading's research outputs online 
1 'Residual diversity estimates' do not correct for sampling bias in

2 palaeodiversity data

3

4 SHORT TITLE: Do not use residuals method

5

6 WORD COUNT: 4,739

7

8 Manabu Sakamoto ${ }^{1}$, Chris Venditti ${ }^{1}$ and Michael J. Benton ${ }^{2}$

9

10 1School of Biological Sciences, University of Reading, Reading, RG6 6AJ, UK

11 2School of Earth Sciences, University of Bristol, Bristol, BS8 1RJ, UK

12

13 EMAIL: m.sakamoto@reading.ac.uk

14

15 
17 1. It is widely accepted that the fossil record suffers from various sampling biases - diversity signals through time may partly or largely reflect the rock record - and many methods have been devised to deal with this problem. One widely used method, the 'residual diversity' method, uses residuals from a modelled relationship between palaeodiversity and sampling (sampling-driven diversity model) as 'corrected' diversity estimates, but the unorthodox way in which these residuals are generated presents serious statistical problems; the response and predictor variables are decoupled through independent sorting, rendering the new bivariate relationship meaningless.

2. Here, we use simple simulations to demonstrate the detrimental consequences of independent sorting, through assessing error rates and biases in regression model coefficients.

3. Regression models based on independently sorted data result in unacceptably high rates of incorrect and systematically, directionally biased estimates, when the true parameter values are known. The large number of recent papers that used the method are likely to have produced misleading results and their implications should be reassessed.

4. We note that the 'residuals' approach based on the sampling-driven diversity model cannot be used to 'correct' for sampling bias, and instead advocate the use of phylogenetic multiple regression models that can include various confounding factors, including sampling bias, while simultaneously accounting for statistical non-independence owing to shared ancestry. Evolutionary dynamics such as speciation are inherently 
a phylogenetic process, and only an explicitly phylogenetic approach will correctly model this process.

\section{KEY WORDS}

44 Palaeodiversity; residuals; modeling; sampling bias; fossil record; independent

45 sorting 
INTRODUCTION

47 It has been well known since the time of Darwin that the fossil record is largely

48 incomplete (Darwin 1859), prompting generations of macroevolutionary

49 researchers to take a cautious approach when interpreting patterns of

50 palaeodiversity through time (Raup 1972; Raup 1976; Raup 1991; Prothero

51 1999; Smith \& McGowan 2007; Alroy 2010b). There have been many attempts to

52 account for this sampling bias (Raup 1972; Raup 1976; Smith \& McGowan 2007;

53 Alroy 2010b), but one approach in particular, often referred to as the 'residual

54 diversity' method, devised by Smith and McGowan (2007) (and modified by

55 Lloyd (2012)), has been widely used (citation count 215 to Aug 2016; Google-

56 Scholar).

57

58

Using regression residuals as data 'corrected' for confounding factors is a widely

59

60

61

62

63

64

65

66

67

68

69

70 used method in biology, social sciences, economics (King 1986; Freckleton

2002), and even in palaeodiversity studies (Raup 1976). However, Smith and

McGowan's (2007) approach differs from these classical residuals approaches in

one key way: the 'residuals' are generated not as regression residuals $(\varepsilon=y-\hat{y})$

from a simple regression of diversity $(y)$ on a proxy of sampling $(x)$, but from " $a$ model in which rock area at outcrop was a perfect predictor of sampled diversity"

(Smith \& McGowan 2007), here referred to as the sampling-driven diversity model (SDDM). The SDDM is constructed as a regression model between $y$ sorted from low to high values $\left(y^{\prime}\right)$ and $x$ sorted from low to high values $\left(x^{\prime}\right)$, where the relationship between these two independently sorted variables $y^{\prime}$ and $x^{\prime}$ is assumed to represent the SDD generating process - though there is no reason to assume as such. 'Residuals' are obtained as the difference between the SDDM 
71 predictions $\hat{y}^{\prime}$ and the observed values $y$, which are then treated as the 'residual

72 diversity estimates' (figure 1).

73

74 However, independently sorting $y$ and $x$ as outlined above decouples a paired,

75 bivariate dataset, and is obviously problematic in statistics. Model fitting on

76 decoupled data (e.g. $y^{\prime}$ and $x^{\prime}$ ) will lead to spurious predictions and 'residuals' as

77 the estimated regression coefficients will be based on a forced (false) linear

78 relationship (figure 1b). However, owing to continued wide use of the SDDM as a

79 preferred method for identifying supposedly 'true' palaeodiversity signals (as

80 recently as (Grossnickle \& Newham 2016)), it appears that this basic statistical

81 concept is somehow overlooked. While it has been suggested that the use of

82 formation counts (the number of fossiliferous geological formations - a

83 mappable unit of rock that represents a particular time and set of environments

84 in a particular location - in a given time interval (Benton et al. 2011)) to 'correct'

85 palaeodiversity time series data is unlikely to be meaningful because of

86 substantial redundancy of the two metrics (Benton et al. 2011; Benton 2015),

87 and a recent study has scrutinized the performance of SDDM residuals in

88 accurately predicting true simulated biodiversity signals (Brocklehurst 2015),

89 the performance of the SDDM itself has never been assessed. Here, we

90 demonstrate the detrimental effects of decoupling data in regression modelling

91 using simple simulations.

92

93

94 MATERIAL AND METHODS 
95 We first generated random deviates, $x$, sampling from a normal distribution $(\mu=$

$960, \sigma=1$ ), at a sample size $n=100$ (see SI for other sample sizes $n=30$ and 1000).

97 We then calculated $y$ using a linear relationship in the form of $y=a+b x+e$,

98 where $a$ is the intercept, $b$ is the slope and $e$ is Gaussian noise. For simplicity, we

99 fixed $a=0.4$ and $b=0.6$, while varying $e\left(\mu_{\mathrm{e}}=0, \sigma_{\mathrm{e}}=0.05,0.1,0.25,0.5\right)-$ other

100 values of $a$ and $b$ should return similar if not identical results (though, $b=1$

101 would be meaningless). Following Smith and McGowan (2007), we sorted $y$ and $x$

102 independently of each other to generate $y^{\prime}$ and $x^{\prime}$, and fitted an ordinary least

103 squares (OLS) regression model to $y^{\prime}$ on $x^{\prime}$ (SDDM). For comparison, we fitted an

104 OLS regression model to $y$ on $x$ in their original paired bivariate relationship (the

105 standard regression model, SRM), the performance of which serves as a

106 benchmark.

108 To test Smith and McGowan's (2007) assertion that the SDDM is indeed "a model

109 in which rock area at outcrop was a perfect predictor of sampled diversity", we

110 evaluated whether the estimated regression coefficients $\alpha$ and $\beta$ significantly

111 differed from the true regression parameters, $a$ and $b$, using a $t$-test. We repeated

112 the procedure over 5000 simulations and calculated the percentage of times the

113 estimated coefficients differed significantly from the true parameters. We would

114 expect about $5 \%$ of the simulations to result in regression coefficients

115 significantly different from the true parameters by chance alone; anything

116 substantially above this threshold would indicate that the model has

117 unacceptably high Type I error rates or falsely rejecting a true null hypothesis,

118 where our null hypothesis is that the SDDM can correctly estimate the 'true'

119 model parameters. 
121 In addition, we tested for bias in the estimated regression slopes, i.e. whether the

122 estimates systematically deviated from the simulation parameter $b=0.6$. The

123 mean of the 5000 slopes was subjected to a $t$-test against a fixed value of 0.6 . If

124 deviations were random, then we would not expect to find any significant

125 differences between the mean slope and thew theoretical value, with all slopes

126 randomly distributed around it.

\section{RESULTS}

130 SRM coefficients were significantly different from the true model parameters in

131 only $\sim 5 \%$ of the 5000 iterations across $\sigma_{\text {e }}$ (figure 2 a; table 1 ; SI), within

132 acceptable levels of randomly detecting a statistical significance. Variation in

133 regression lines across 5000 iterations are distributed randomly about the

134 simulated line (figure 3a), with no significant difference between the mean

135 regression slope and the simulation parameter $b=0.6$ (table 2; SI). In contrast,

136 SDDM coefficients were significantly different from the true parameters (figure

$1372 \mathrm{~b}$ ) at a rate much higher than the conventionally accepted $5 \%$ (table $1 ; \mathrm{SI}$ ). The

138 mean slope of the regression models significantly differed from the simulation

139 parameter $b$, in a systematically and directional manner (figure $3 \mathrm{~b}$; table 2 ; SI) -

140 SDDM regression coefficients are not only incorrect but grossly misleading. This

141 systematic bias increases with increased noise in the data (table 2) - the more

142 noise there is in the data, the more positive the relationship between $y^{\prime}$ and $x^{\prime}$

143 becomes. 


\section{DISCUSSION}

147 By establishing "a model in which rock area at outcrop was a perfect predictor of 148 sampled diversity", Smith and McGowan (2007) attempted to create a sampling149 driven diversity model. However, their SDDM is not based on any hypothesized 150 or empirical relationship between diversity and sampling, or formulated from

151 first principles. This is in contrast to other well-formulated biological models

152 such as various scaling models where the parameter of interest (i.e. scaling 153 coefficient or the slope of the bivariate relationship) is founded on first-principle 154 theories, e.g. the $2 / 3$ rule for the scaling of area with mass. Rather, the SDDM is 155 based on the assumption that $y^{\prime}$ and $x^{\prime}(y$ and $x$ sorted independently of each 156 other) form the expected theoretical bivariate relationship between $y$ and $x$, 157 which this study shows to be incorrect (figures 2, 3), as one would expect since 158 there is no reason to assume such a thing.

160 A further and perhaps more serious problem with using a forced pairing of $y^{\prime}$ and $161 x^{\prime}$ is that each data point (pair of $y^{\prime}{ }_{i}$ and $x^{\prime}$ i) does not represent a natural pairing 162 and has no meaning; the new pairing is actually $y_{\mathrm{i}}$ and $x_{\mathrm{j}}$, where the ith and jth 163 orders are independent of each other. For instance, using the marine generic 164 diversity and rock area data of Smith and McGowan (2007) (figure 4), the lowest 165 marine generic diversity is in the Cambrian, Tommotian Stage (529 - 521 million 166 years ago [Ma]; genus count = 309), while the smallest marine rock outcrop area 167 (after removing 0 valued data (Smith \& McGowan 2007)) is from the Early 168 Permian, Asselian/Sakmarian Stage (299 - 290 Ma; rock area = 1). Similarly, the 169 highest diversity is recorded for the Pliocene $(5.3-2.58 \mathrm{Ma}$; genus count $=3911)$ 
while the largest rock area is found in the Cenomanian (100 - 94 Ma; rock area = 373). These two extreme points alone demonstrate that the paired diversity and rock area values are millions of years apart, and are independent of each other (figure 4).

This may be obvious, but independently sorting $y$ and $x$ has serious statistical consequences. For instance, in Smith and McGowan's (2007) data, $\log _{10}$ marine generic diversity has no significant relationship with $\log _{10}$ rock area in their original paired bivariate data (figure 4; $r^{2}=0.0398 ; p=0.0979$ ), but once sorted, has a significantly strong positive relationship with $\log _{10}$ rock area sorted independently of $\log _{10}$ diversity (figure $4 ; r^{2}=0.903 ; p<0.001$ ). This general pattern is true in at least two more datasets (Benson et al. 2010; Benson \& Upchurch 2013) (figures S1 and S2). The independent sorting procedure has forced a strong but false linear relationship between two variables that otherwise do not show any significant (or if significant, a very weak) relationship. In fact, two randomly generated deviates (e.g. sampled from a normal distribution) that have no relationship with each other (figure 5a), once sorted independently from lowest to highest will inevitably have a significant and strong relationship ( $r^{2}=\sim 1$; figure $5 b$ ). Perhaps more detrimental, is the fact that the independently sorted bivariate relationship will always be strongly positive - a simulated negative relationship between $x$ and $y$ (figure 5c) will have a strong and positive relationship once they are sorted independently (figure 5d). 
194 In some clades (namely Mesozoic dinosaurs), diversity measures can have very

195 strongly positive relationships with some sampling metrics, such as geological

196 formation counts $\left(\beta=0.868 ; r^{2}=0.85 ; p<0.001\right.$ (Barrett, McGowan \& Page

1972009 ) ) or fossil collection counts $\left(\beta=0.865 ; r^{2}=0.79 ; p<0.001\right.$ (Butler et al.

198 2011)), which would justify correcting for such confounding factors, if the

199 sampling metrics were indeed non-redundant with diversity (Benton et al. 2011;

200 Benton et al. 2013). However, even in such cases, it does not change the fact that

201 the modelled relationship obtained from the SDDM will still be systematically

202 biased (figure 3), and alternative methods should be considered.

203

204 It is problematic to stipulate that this forced relationship is the 'true' relationship

205 between sampled palaeodiversity and the rock record. Our simulations show

206 that regression models fitted on independently sorted data have unacceptably

207 high Type I error rates when the data generation processes are known, meaning

208 that Smith and McGowan's (2007) approach is not statistically viable. In

209 particular, that the slopes are incorrectly estimated at very high rates $(\sim 100 \%$

210 when $\sigma_{\mathrm{e}}=0.5$ ) has severe consequences in that SDDM predictions are

211 systematically biased (figures $2 \mathrm{~b}, 3 \mathrm{~b}$ ), leading to erroneous 'residuals'.

212 Inferences made from such problematic 'residuals' (Smith \& McGowan 2007;

213 Barrett, McGowan \& Page 2009; Benson et al. 2010; Butler et al. 2011; Benson \&

214 Upchurch 2013) will inevitably be misleading (Brocklehurst 2015), lacking any

215 biological or geological meaning.

217 Given our simulations, we strongly recommend against using the SDDM

218 approach in modelling the relationship between palaeodiversity and rock record 
data; the standard regression using unsorted data is a sensible option. However,

220 using the residuals of a regression model as data for subsequent analyses has

221 also long been known to introduce biased statistical estimates (King 1986;

222 Freckleton 2002). Successive series of modelling removes variance and degrees

223 of freedom from subsequent model parameter estimation, so the final models

224 and statistical analyses do not account for the removed errors appropriately

225 (King 1986). Instead, one can directly model the confounding effects along with

226 effects of interest (e.g. environment, climate, etc) through multiple regressions

227 (OLS, GLMs or generalized least squares [GLS]). In the context of palaeodiversity

228 studies, one can fit a multiple regression model using some diversity metric as

229 the response variable and sampling proxy as a confounding covariate, alongside

230 additional predictor variables such as sea level, temperature, etc. The resulting

231 model coefficients for the environmental predictors would be the effects of

232 interest after accounting for the undesired effects of rock availability. Since

233 diversity measures are frequently taken as counts, it is advisable to use models

234 that appropriately account for errors in count data, such as the Poisson or

235 negative binomial models (O'Hara \& Kotze 2010). Whether or not to include time

236 series terms (e.g. autoregressive [AR] terms) depends on the level of serial

237 autocorrelation in the time series data and on sample size; palaeontological time

238 series tend to be short, with 30 time bins or fewer being fairly typical (Mesozoic

239 dinosaurs only span a maximum of 26 geological stages (Butler et al. 2011;

240 Benson \& Mannion 2012)), in which case complex models face the risks of over-

241 parameterisation. Model selection procedures using the Akaike Information

242 Criterion (Akaike 1973) or similar indices can help make this decision (Burnham

243 \& Anderson 2002). However, we do not lightly advocate the use of time series 
244 modelling, especially if the dependent variable, sampled diversity, is in the form

245 of counts, in which case appropriate time series methods are severely under-

246 developed (but see generalised linear autoregressive moving average [GLARMA]

247 models (Dunsmuir \& Scott 2015) or Poisson exponentially weighted moving

248 average [PEWMA] models (Brandt et al. 2000)), but more importantly since

249 there are more appropriate alternative methods, i.e. phylogenetic approaches

250 (Sakamoto, Benton \& Venditti 2016).

251

252 Fundamentally, macroevolutionary studies aim to increase our understanding of

253 evolutionary processes (speciation and extinction through time), rather than the

254 resulting patterns or phenomena (sampled diversity, e.g. richness). Thus, we

255 should seek to characterize the process using biologically meaningful and

256 interpretable models instead of describing the patterns. Further, simply

257 exploring error in the fossil record in itself seems rather fruitless because

258 uncertainty depends on the questions being posed; palaeontological studies of

259 macroevolution should be no different than other statistical approaches in the

260 natural sciences in that uncertainty is assessed while exploring the phenomena

261 of interest (Benton 2015). Explicitly phylogenetic approaches (e.g. (Lloyd et al.

262 2008; Didier, Royer-Carenzi \& Laurin 2012; Stadler 2013; Stadler et al. 2013;

263 Sakamoto, Benton \& Venditti 2016) offer the best and most appropriate means to

264 tackle questions of evolutionary processes. Especially when extrinsic causal

265 mechanisms for changes in biodiversity are tested using regression models,

266 ignoring phylogeny is in serious violation of statistical independence

267 (Felsenstein 1985; Harvey \& Pagel 1991). It is also worth noting that

268 subsampling approaches (e.g. Alroy's SQS (Alroy 2010a; Alroy 2010b; Alroy 

2010c)) are gaining wide popularity as modern methods to account for sampling

270 bias, they are not without problems (Hannisdal et al. 2016), and certainly do not

271 take shared ancestry described by phylogeny into account, thus also suffering 272 statistical non-independence (Felsenstein 1985; Harvey \& Pagel 1991), and can 273 frequently result in incorrect interpretation of the data. For instance, while 274 recent studies using binned time series approaches (including SDDM and SQS)

275 have led to mixed conclusions regarding the long-term demise of dinosaurs 276 before their final extinction at the Cretaceous-Paleogene (K-Pg) boundary 66 277 million years ago (Ma) (Barrett, McGowan \& Page 2009; Lloyd 2012; Brusatte et 278 al. 2015), an explicitly phylogenetic Bayesian analysis has strongly suggested 279 that dinosaurs were indeed in a long-term decline tens of millions of years prior 280 to the K-Pg mass extinction event, in which speciation rate was exceeded by 281 extinction rate and dinosaurs were increasingly incapable of replacing extinct 282 taxa with new ones (Sakamoto, Benton \& Venditti 2016). Such evolutionary 283 dynamics cannot be identified using time-binned (tabulated) data. Phylogenetic 284 mixed modelling approaches (Hadfield 2010) further allow the incorporation of 285 confounding variables such as sampling but also environmental effects 286 (Sakamoto, Benton \& Venditti 2016). Therefore, in order to advance our 287 understanding of the evolutionary dynamics of biodiversity, speciation and 288 extinction through time (or the underlying process generating the observed 289 patterns in sampled diversity, e.g. taxon richness), while accounting for sampling 290 and phylogenetic non-independence, it is imperative that we have an abundance 291 of large-scale comprehensive phylogenetic trees of fossil (and extant) taxa. 
295 We thank Jo Baker, Ciara O’Donovan and Henry Ferguson-Gow for discussion

296 and insightful comments. We also thank Neil Brocklehurst and Michel Laurin for

297 reviewing this manuscript and providing helpful commentary. We have no

298 conflicts of interest.

DATA ACCESSIBILITY

This manuscript does not include data.

FUNDING

MS and CV are funded by Leverhulme Trust Research Project Grant RPG-2013-

Standard Grant NE/I027630/1.

\section{REFERENCES}

Akaike, H. (1973) Information theory and an extension of the maximum likelihood principle. 2nd International Symposium on Information Theory (eds B.N. Petrov \& F. Csaki), pp. 267-281. Akademiai Kiado, Budapest.

Alroy, J. (2010a) Fair sampling of taxonomic richness and unbiased estimation of origination and extinction rates. Quantitative methods in paleobiology. Paleontological Society Papers, 16, 55-80.

Alroy, J. (2010b) Geographical, Environmental and Intrinsic Biotic Controls on Phanerozoic Marine Diversification. Palaeontology, 53, 1211-1235.

Alroy, J. (2010c) The Shifting Balance of Diversity Among Major Marine Animal Groups. Science, 329, 1191-1194.

Barrett, P.M., McGowan, A.J. \& Page, V. (2009) Dinosaur diversity and the rock record. Proceedings Of The Royal Society B-Biological Sciences, 276, 2667 2674.

Benson, R.B.J., Butler, R.J., Lindgren, J. \& Smith, A.S. (2010) Mesozoic marine tetrapod diversity: mass extinctions and temporal heterogeneity in 
geological megabiases affecting vertebrates. Proceedings Of The Royal Society B-Biological Sciences, 277, 829-834.

Benson, R.B.J. \& Mannion, P.D. (2012) Multi-variate models are essential for understanding vertebrate diversification in deep time. Biology Letters, $\mathbf{8}$, 127-130.

Benson, R.B.J. \& Upchurch, P. (2013) Diversity trends in the establishment of terrestrial vertebrate ecosystems: Interactions between spatial and temporal sampling biases. Geology, 41, 43-46.

Benton, M.J. (2015) Palaeodiversity and formation counts: redundancy or bias? Palaeontology, 58, 1003-1029.

Benton, M.J., Dunhill, A.M., Lloyd, G.T. \& Marx, F.G. (2011) Assessing the quality of the fossil record: insights from vertebrates. Comparing the Geological and Fossil Records: Implications for Biodiversity Studies, 358, 63-94.

Benton, M.J., Ruta, M., Dunhill, A.M. \& Sakamoto, M. (2013) The first half of tetrapod evolution, sampling proxies, and fossil record quality. Palaeogeography Palaeoclimatology Palaeoecology, 372, 18-41.

Brandt, P.T., Williams, J.T., Fordham, B.O. \& Pollins, B. (2000) Dynamic modeling for persistent event-count time series. American Journal of Political Science, 44, 823-843.

Brocklehurst, N. (2015) A simulation-based examination of residual diversity estimates as a method of correcting for sampling bias. Palaeontologia Electronica, 18.

Brusatte, S.L., Butler, R.J., Barrett, P.M., Carrano, M.T., Evans, D.C., Lloyd, G.T., Mannion, P.D., Norell, M.A., Peppe, D.J., Upchurch, P. \& Williamson, T.E. (2015) The extinction of the dinosaurs. Biological Reviews, 90, 628-642.

Burnham, K.P. \& Anderson, D.R. (2002) Model selection and multimodel inference: a practical information - theoretical approach, 2nd edn. Springer, New York.

Butler, R.J., Benson, R.B.J., Carrano, M.T., Mannion, P.D. \& Upchurch, P. (2011) Sea level, dinosaur diversity and sampling biases: investigating the 'common cause' hypothesis in the terrestrial realm. Proceedings Of The Royal Society B-Biological Sciences, 278, 1165-1170.

Darwin, C. (1859) On the Origin of Species by Means of Natural Selection, or the Preservation of Favoured Races in the Struggle for Life, First Edition edn., London, UK.

Didier, G., Royer-Carenzi, M. \& Laurin, M. (2012) The reconstructed evolutionary process with the fossil record. Journal of Theoretical Biology, 315, 26-37.

Dunsmuir, W.T.M. \& Scott, D.J. (2015) The glarma Package for ObservationDriven Time Series Regression of Counts. Journal of Statistical Software, 67, 1-36.

Felsenstein, J. (1985) Phylogenies and the Comparative Method. American Naturalist, 125, 1-15.

Freckleton, R. (2002) On the misuse of residuals in ecology: regression of residuals vs. multiple regression. (vol 71, pg 542, 2002). Journal of Animal Ecology, 71, 722-722.

Grossnickle, D.M. \& Newham, E. (2016) Therian mammals experience an ecomorphological radiation during the Late Cretaceous and selective extinction at the K-Pg boundary. Proceedings of the Royal Society of London B: Biological Sciences, 283. 
Hadfield, J.D. (2010) MCMC methods for multi-response Generalized Linear Mixed Models: The MCMCglmm R Package. Journal of Statistical Software, 33, 1-22.

Hannisdal, B., Haaga, K.A., Reitan, T., Diego, D. \& Liow, L.H. (2016) Common species link global ecosystems to climate change. bioRxiv, 043729.

Harvey, P.H. \& Pagel, M.D. (1991) The comparative method in evolutionary biology. Oxford University Press.

King, G. (1986) How Not to Lie with Statistics - Avoiding Common Mistakes in Quantitative Political-Science. American Journal of Political Science, 30, 666-687.

Lloyd, G.T. (2012) A refined modelling approach to assess the influence of sampling on palaeobiodiversity curves: new support for declining Cretaceous dinosaur richness. Biology Letters, 8, 123-126.

Lloyd, G.T., Davis, K.E., Pisani, D., Tarver, J.E., Ruta, M., Sakamoto, M., Hone, D.W.E., Jennings, R. \& Benton, M.J. (2008) Dinosaurs and the Cretaceous Terrestrial Revolution. Proceedings Of The Royal Society B-Biological Sciences, 275, 2483-2490.

O'Hara, R.B. \& Kotze, D.J. (2010) Do not log-transform count data. Methods in Ecology and Evolution, 1, 118-122.

Prothero, D. (1999) Fossil record. Encyclopedia of paleontology (ed. R. Singer). Fitzroy Dearbon Publishers, Chicago, USA.

Raup, D.M. (1972) Taxonomic Diversity during the Phanerozoic. Science, 177, 1065-1071.

Raup, D.M. (1976) Species Diversity in the Phanerozoic: An Interpretation. PALEOBIOLOGY, 2, 289-297.

Raup, D.M. (1991) Extinction: bad genes or bad luck? W. W. Norton, New York.

Sakamoto, M., Benton, M.J. \& Venditti, C. (2016) Dinosaurs in decline tens of millions of years before their final extinction. Proceedings of the National Academy of Sciences, 113, 5036-5040.

Smith, A.B. \& McGowan, A.J. (2007) The shape of the phanerozoic marine palaeodiversity curve: How much can be predicted from the sedimentary rock record of western Europe? Palaeontology, 50, 765-774.

Stadler, T. (2013) Recovering speciation and extinction dynamics based on phylogenies. Journal Of Evolutionary Biology, 26, 1203-1219.

Stadler, T., Kuhnert, D., Bonhoeffer, S. \& Drummond, A.J. (2013) Birth-death skyline plot reveals temporal changes of epidemic spread in HIV and hepatitis $\mathrm{C}$ virus (HCV). Proceedings Of The National Academy Of Sciences of The United States Of America, 110, 228-233. 
414 SUPPORTING INFORMATION

415 SI-text. Supporting information and results pertaining to the effects of sample 416 size (Tables S1 and S2) as well as examples of discrepancies between original

417 paired bivariate relationship and the independently sorted relationship from the 418 literature (Figs S1 and S2). 
TABLES

420 Table 1. Type I error rates (\%) for SRM (Standard Regression Model) and SDDM 421 (Sampling-Driven Diversity Model) estimates (intercept $\alpha$ and slope $\beta$ ) across 422 residual error $\left(\sigma_{\mathrm{e}}\right)$.

423

\begin{tabular}{|r|rr|crr|}
\hline \multicolumn{1}{|c|}{$\boldsymbol{\sigma}_{\mathrm{e}}$} & \multicolumn{2}{|c|}{ SRM } & \multicolumn{2}{c|}{ SDDM } \\
\hline 0.05 & $\boldsymbol{\alpha}$ & $\boldsymbol{B}$ & $\boldsymbol{\alpha}$ & \multicolumn{1}{c|}{$\boldsymbol{~}$} \\
0.10 & 5.34 & 4.90 & 26.1 & 28.5 \\
0.25 & 4.84 & 4.92 & 40.2 & 48.4 \\
0.50 & 4.82 & 4.78 & 57.3 & 91.3 \\
\hline
\end{tabular}

424 
425 Table 2. $t$-test results between mean regression slopes of 5000 iterations and the 426 theoretical slope $b=0.6$, for SRM (Standard Regression Model) and SDDM 427 (Sampling-Driven Diversity Model) across residual error $\left(\sigma_{\mathrm{e}}\right)$.

428

\begin{tabular}{|r|rrr|rrr|}
\hline \multirow{2}{*}{$\sigma_{\mathbf{e}}$} & \multicolumn{3}{|c|}{ SRM } & \multicolumn{3}{c|}{ SDDM } \\
& mean-slope & $\boldsymbol{t}$-value & $\boldsymbol{p}$-value & mean-slope & $\boldsymbol{t}$-value & $\boldsymbol{p}$-value \\
\hline 0.05 & 0.6 & 1.230 & 0.220 & 0.602 & 20.9 & 0 \\
0.10 & 0.6 & -1.790 & 0.073 & 0.607 & 46.0 & 0 \\
0.25 & 0.6 & -0.042 & 0.967 & 0.646 & 131.0 & 0 \\
0.50 & 0.6 & 0.685 & 0.493 & 0.775 & 244.0 & 0 \\
\hline
\end{tabular}


431 residuals (a) and the SDDM 'residuals' (c).
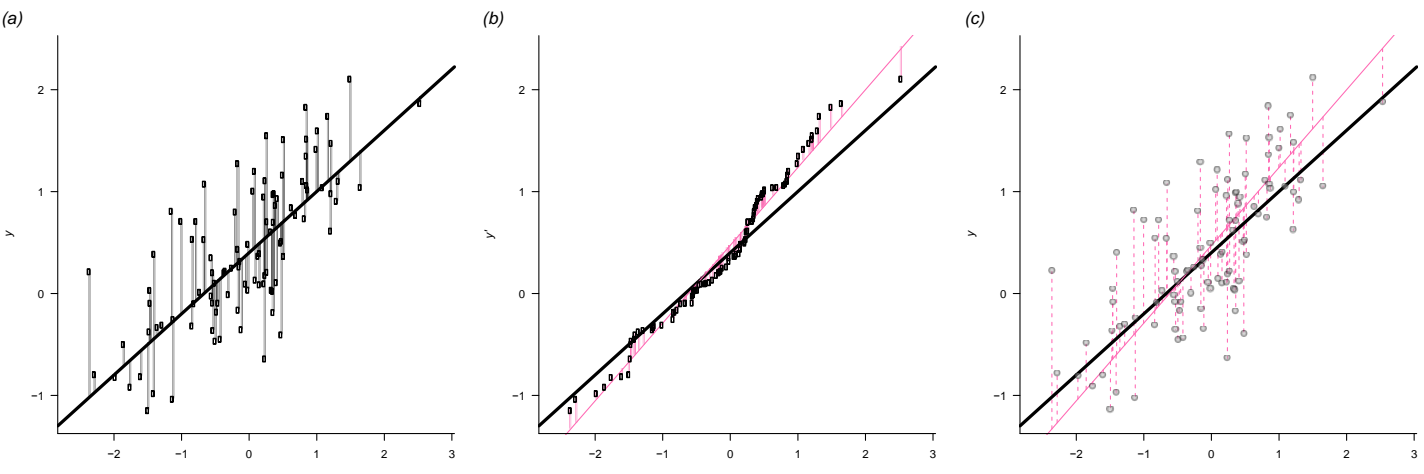

Figure 1. Procedure for generating 'residuals' from a sampling-driven diversity model. (a) A paired, bivariate dataset $x$ (sampling proxy) and $y$ (sampled diversity) was simulated so that $x$ is randomly drawn from a normal distribution $(\mu=0, \sigma=1)$ and $y$ is calculated as $y=a+b x+e$ where $a=0.4, b=0.6$ and $e$ is noise $(\mu=0, \sigma=0.5)$. The thick black line is the expected relationship $Y=a+b x$.

Vertical lines represent the true residuals or deviations in $y$ from the thick line. (b) Following Smith and McGowan (2007) $x$ and $y$ are sorted from low to high values independent of each other ( $x^{\prime}$ and $y^{\prime}$ respectively), and an ordinary least squares (OLS) regression model (pink line) is fitted to $y^{\prime}$ on $x^{\prime}$. Despite the pink line supposedly representing the data generating process, it is clear that it is not a good estimator of the true known generating process, the thick line. (c) The OLS model from (b) is used as the sampling-driven diversity model (SDDM) or the expected relationship between $y$ and $x$, from which 'residuals' are computed as the deviations in $y$ from the pink line (vertical pink dotted lines). It is immediately clear that there is a substantial difference between the true 
(a)

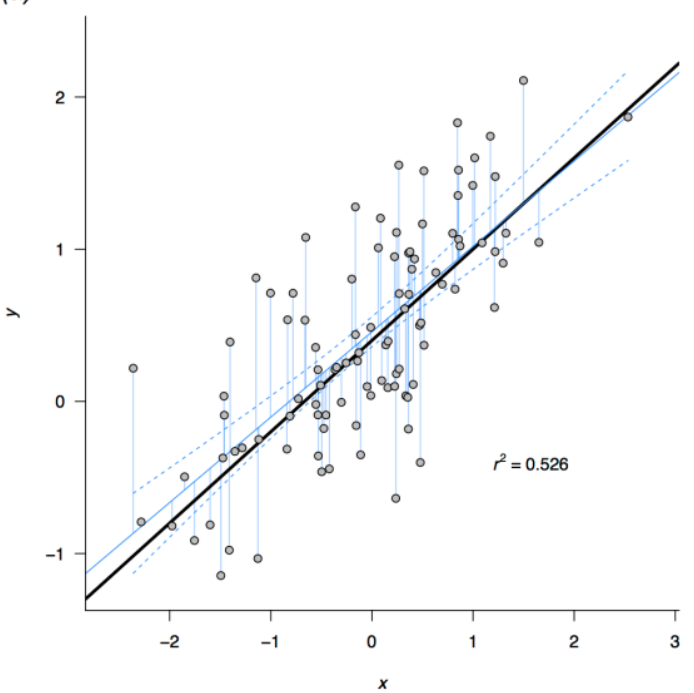

(b)

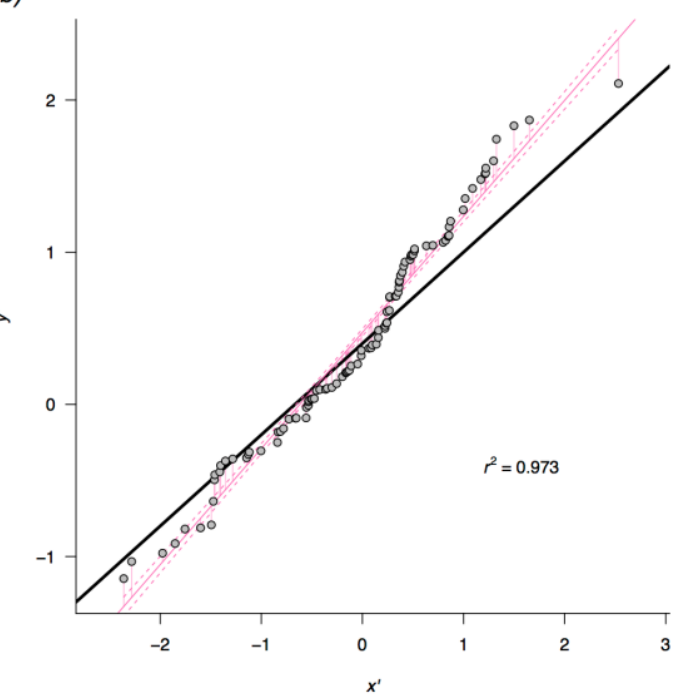

449

Figure 2. Regression modelling on a decoupled bivariate dataset fails to estimate the simulation slope parameter. ( $a$ ) A bivariate dataset $(y$ and $x$ ) was generated so as to follow a theoretical relationship (thick line) with intercept $a=0.4$, slope $b=0.6$ and noise $\left(e\left[\mu_{\mathrm{e}}=0, \sigma_{\mathrm{e}}=0.5\right]\right)$. The best-fit regression line (blue) is not significantly different from the theoretical line (dashed 95\% confidence intervals encompass the thick line; see table 1 for Type I error rates over 5000 simulations), with $y$ and $x$ forming a moderately strong relationship $\left(r^{2}=0.526\right)$ appropriate for the degree of $e$ modelled. Regression model residuals (vertical lines) show no structure, as expected. (b) The bivariate data in $(a)$ were sorted independently of each other $\left(y^{\prime}\right.$ and $\left.x^{\prime}\right)$, to which a regression model was fitted. The best-fit sampling-driven diversity model (SDDM) regression line (pink) deviates strongly from the theoretical relationship (dashed 95\% confidence intervals do not encompass the thick line; table 1 ), and $y^{\prime}$ and $x^{\prime}$ form a very strong (but false) linear relationship $\left(r^{2}=0.973\right)$. Regression residuals (vertical lines) show clear structure. One pair of model comparison out of 5000 simulations is shown. 
(a)

a)

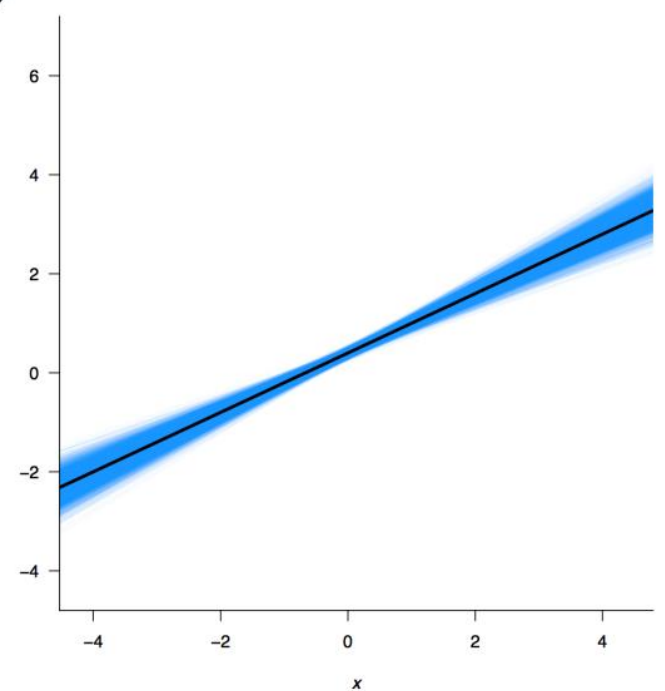

(b)

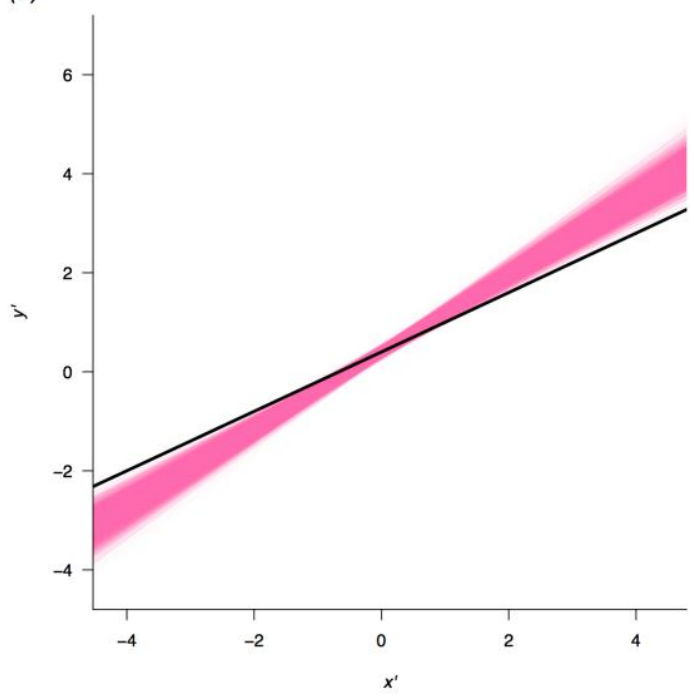

467

468 Figure 3. SDDM regression predictions are systematically biased. (a) Standard regression lines (blue) for 5000 simulated datasets at $\sigma_{\mathrm{e}}=0.5$ deviate randomly around the theoretical relationship (thick line) with the mean slope showing no

471 significant difference from the theoretical slope $b=0.6$ (table 2). (b) SDDM

472 regression lines on decoupled datasets (pink) deviate systematically away from

473 the theoretical relationship (thick line), with a significant difference between the 474 mean regression slope and the theoretical slope (table 2). 
(a)

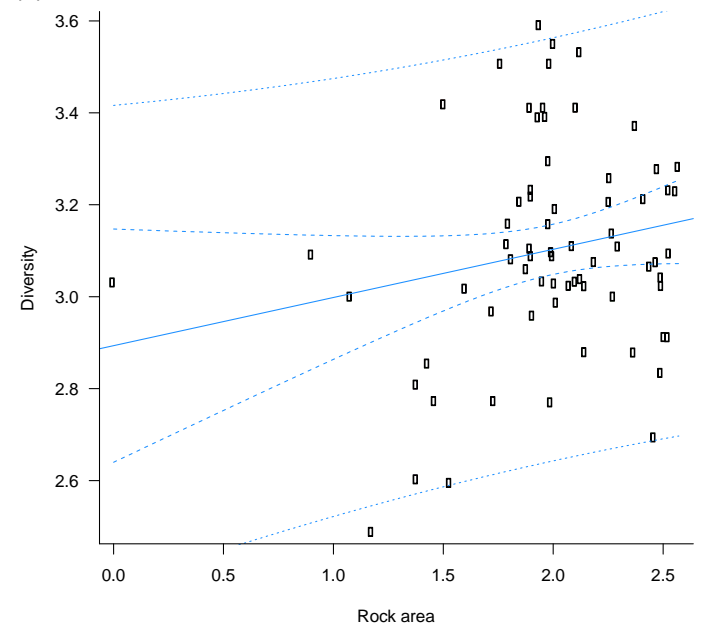

(b)

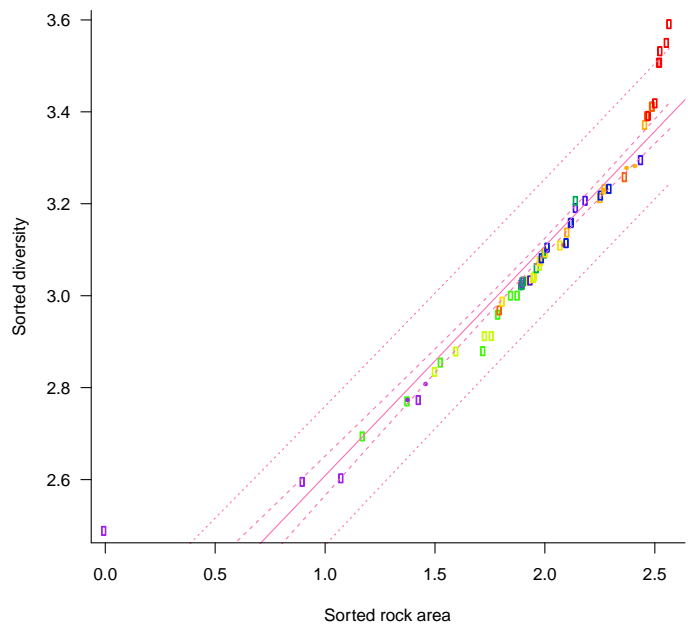

476

477 Figure 4. The difference between the original paired, bivariate relationship (a)

478 and the forced, false relationship (b) shown using the data from Smith and

479 McGowan (2007). Log-transformed marine generic diversity has a non-

480 significant and weak relationship with log-transformed rock area $\left(\beta=0.105 ; r^{2}=\right.$

$4810.0398 ; p=0.0979 ; \mathrm{a})$. However, once diversity and rock area are sorted

482 independently of each other following Smith and McGowan (2007), then the

483 relationship becomes significant and strong $\left(\beta=0.499 ; r^{2}=0.903 ; p<0.001 ; b\right)$.

484 Points are coloured according to their geological age with cooler colours on the

485 older and warmer colours on the younger ends of the time scale. Filled and

486 outline colours in (b) correspond to the ages of the rock record and diversity

487 respectively, and demonstrate visually the mismatch between $y^{\prime}$ and $x^{\prime}$. Dashed

488 lines are confidence intervals, while dotted lines are prediction intervals. 
(a)

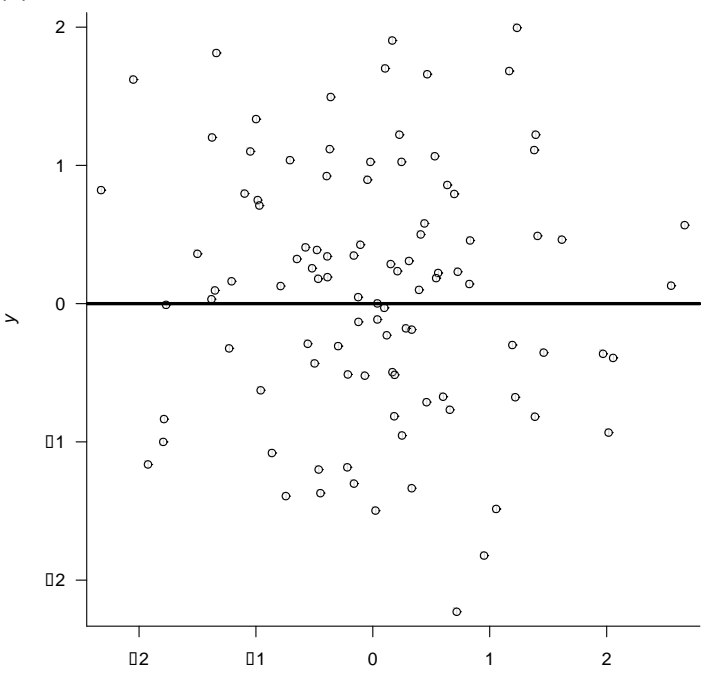

(c)

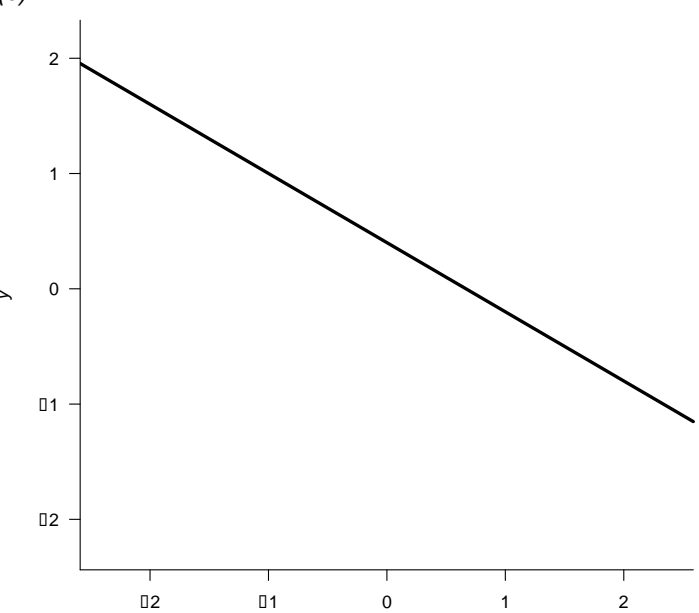

(b)

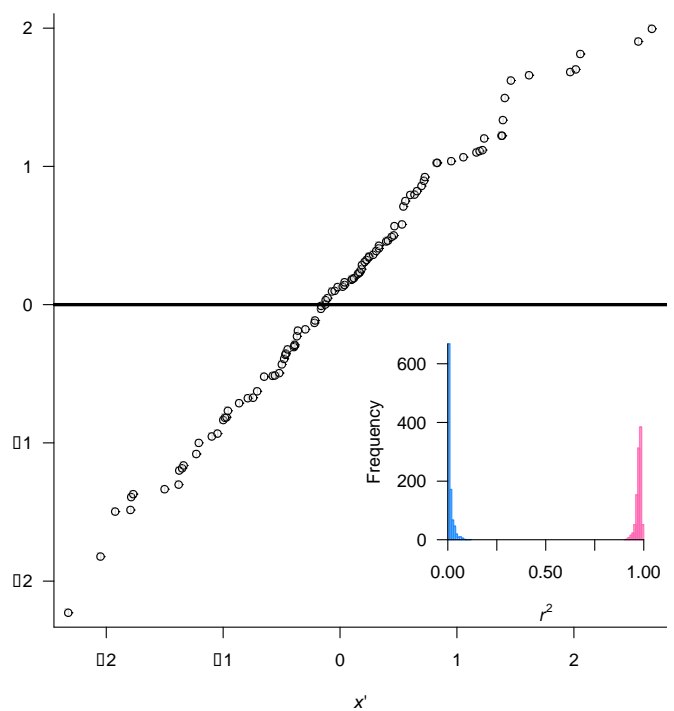

(d)

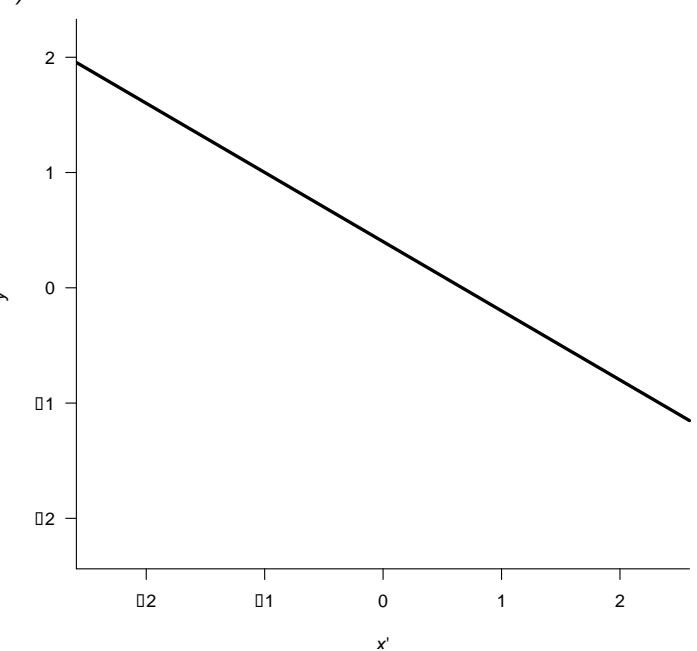

491 Figure 5. Independently sorting any two variables results in a forced positive relationship. (a) Two randomly generated variables $y$ and $x$ show no significant relationships across 1000 simulations, with the slopes of the regression lines

494 (blue) distributed around the expected slope of zero. (b) When regression models are fitted on independently sorted datasets $\left(y^{\prime}\right.$ and $\left.x^{\prime}\right)$, estimated slopes are significantly different from the expected value of zero, and result in a strong

497 positive relationship $\left(r^{2}=\sim 1\right.$; inset pink) despite the unrelated nature of the 498 original datasets $\left(r^{2}=\sim 0\right.$; inset blue). ( $c$ ) A bivariate dataset ( $y$ and $x$ ) was generated so as to follow a theoretical relationship (thick line) with intercept $a=$ 0.4 , slope $b=-0.6$ and noise $\left(e\left[\mu_{\mathrm{e}}=0, \sigma_{\mathrm{e}}=0.5\right]\right)$. Standard regression lines (blue) 
501 deviate randomly around the theoretical relationship with the mean slope

502 showing no significant difference from the theoretical slope $b=-0.6$. (d) However

503 once sorted independently, regression lines (pink) deviate systematically away

504 from the theoretical relationship, with all estimated slopes being positive. Thus

505 SDDM slope estimates are systematically and directionally biased.

506 